\title{
AVALIAÇÃO DO EFEITO DA TEMPERATURA, PH E GRANULOMETRIA DO ADSORVENTE NA ADSORÇÃO DO CORANTE AZUL REATIVO 5G
}

\author{
Pricila Marin ${ }^{1}$ \\ Carlos Eduardo Borba ${ }^{2}$ \\ Aparecido Nivaldo Módenes ${ }^{3}$ \\ Silvia Priscila Dias de Oliveira ${ }^{4}$ \\ Nathalia Passaia 5 \\ Lucas S. Figueiredo ${ }^{6}$
}

\begin{abstract}
Resumo: Neste trabalho foram realizados experimentos para avaliação do efeito do $\mathrm{pH}$ da solução $(1-12)$, da temperatura do sistema $\left(20,30,40\right.$ e $\left.50^{\circ} \mathrm{C}\right)$ e do diâmetro das partículas de adsorvente $(0,055 ; 0,063 ; 0,090 \mathrm{~cm})$ na remoção do corante Azul Reativo $5 \mathrm{G}$ utilizando o adsorvente comercial Dowex ${ }^{\mathrm{TM}}$ Optipore $^{\mathrm{TM}} \mathrm{SD}-2$. Os experimentos foram realizados em sistema fechado e batelada colocando-se $50 \mathrm{~mL}$ de solução de corante com concentração de $150 \mathrm{mg} \mathrm{L}^{-1}$ em contato com $0,005 \mathrm{~g}$ de adsorvente sob agitação constante $(120 \mathrm{rpm})$ por um período de 96 horas. Além disto, foi determinado o ponto de carga zero do adsorvente $\left(\mathrm{pH}_{\mathrm{PCZ}}\right)$ pelo método de titulação potenciométrica. As condições experimentais que apresentaram os melhores resultados em relação à quantidade de corante adsorvido foram: $\mathrm{pH}$ da solução igual a 2, temperatura do sistema de $30^{\circ} \mathrm{C}$ e partículas de adsorvente com diâmetro entre 0,01 e $0,12 \mathrm{~cm}$. Os valores obtidos para os parâmetros termodinâmicos indicam que o processo é exotérmico, espontâneo e possui afinidade entre adsorvente/adsorvato. $\mathrm{O} \mathrm{pH} \mathrm{PCZ}_{\mathrm{PC}}$ do adsorvente ficou entre $4 \mathrm{e} 10$. Em todos os testes realizados, o adsorvente Dowex ${ }^{\mathrm{TM}}$ Optipore $^{\mathrm{TM}}$ SD-2 apresentou alta capacidade de adsorção do corante Azul Reativo 5G (superior a $100 \mathrm{mg} \mathrm{g}^{-1}$ ) sendo que nas melhores condições, entre as avaliadas, a capacidade de adsorção foi superior a $230 \mathrm{mg} \mathrm{g}^{-1}$. Desta forma, o adsorvente estudado apresenta grande potencial para ser utilizado em sistemas de tratamento de efluentes têxteis.
\end{abstract}

Palavras-chave: Corante Azul Reativo 5G, adsorção, Dowex ${ }^{\mathrm{TM}}$ Optipore ${ }^{\mathrm{TM}}$ SD-2.

Abstract: In this work, experiments were performed to evaluate the effect of solution $\mathrm{pH}(1-12)$, system temperature $\left(20,30,40\right.$ and $\left.50{ }^{\circ} \mathrm{C}\right)$ and the particle diameter of the adsorbent $(0,055 ; 0,063 ; 0,090 \mathrm{~cm})$ in the removal of Reactive Blue $5 \mathrm{G}$ using commercial adsorbent Dowex ${ }^{\mathrm{TM}}$ Optipore ${ }^{\mathrm{TM}} \mathrm{SD}-2$. The experiments were carried out in a closed and batch system placing $50 \mathrm{~mL}$ of the dye solution with a concentration of $150 \mathrm{mg} \mathrm{L}$ 1 in contact with $0.005 \mathrm{~g}$ of adsorbent under constant agitation $(120 \mathrm{rpm})$ for a period of 96 hours. Moreover, was determined the point of zero charge $\left(\mathrm{pH}_{\mathrm{PCZ}}\right)$ of the adsorbent by potentiometric titration method. The experimental conditions that showed the best results in relation to amount of dye adsorbed were: solution $\mathrm{pH}$ equal to 2 , the system temperature of $30^{\circ} \mathrm{C}$ and adsorbent particles with a diameter between 0.01 to $0.12 \mathrm{~cm}$. The values obtained for the thermodynamic parameters indicate that the process is exothermic, spontaneous and has adsorbent/adsorbate affinity. The adsorbent $\mathrm{pH}_{\mathrm{PCZ}}$ was between 4 and 10. In all tests, the adsorbent Dowex ${ }^{\mathrm{TM}}$ Optipore $^{\mathrm{TM}} \mathrm{SD}-2$ showed high adsorption capacity of dye Reactive Blue 5G (higher than $100 \mathrm{mg} \mathrm{g}^{-1}$ ) whereas on the best conditions among the evaluated, the adsorption capacity was higher than $230 \mathrm{mg} \mathrm{g}^{-1}$. Thus, the adsorbent studied presents great potential to be used in wastewater treatment systems for textile effluents.

${ }^{1}$ Programa de Pós-graduação Stricto Sensu em Engenharia Química da Universidade Estadual do Oeste do Paraná. E-mail: pricila_marin@hotmail.com 
Key-words: Reactive Blue dye 5G, adsorption, Dowex ${ }^{\mathrm{TM}}$ Optipore ${ }^{\mathrm{TM}}$ SD-2.

\section{INTRODUÇÃO}

Efluentes contendo corantes são hoje uma das grandes preocupações ambientais. A presença de corantes nos corpos hídricos afeta a coloração da água resultando na redução da penetração de luz (Alkan et al., 2008), diminuindo assim a eficiência da fotossíntese em plantas aquáticas e, portanto, gerando um efeito adverso no seu crescimento (AlDegs et al., 2000).

As indústrias têxteis são as principais responsáveis pela presença de corantes no meio ambiente, visto que, mais de $60 \%$ dos corantes produzidos mundialmente são utilizados no beneficiamento têxtil e, deste total, em consequência das perdas do processo, mais da metade acaba sendo despejada em corpos d'água sem nenhum tipo de tratamento (Khadhraoui et al., 2009). Atualmente, mais de $90 \%$ dos corantes utilizados na indústria e comércio são substâncias sintéticas (Tríplice cor, 2011) e, destes, um dos mais comuns é o corante reativo.

O corante Azul Reativo 5G, produzido e comercializado pela Texpal Indústria Química, apresenta excelente comportamento tintório e boa penetração, sendo amplamente utilizado em processos de lavanderia industrial e tingimento têxtil (Texpal, 2005). É composto por um grupo cromóforo do tipo azo, responsável pela cor, e um grupo sulfonato, responsável pela solubilidade e caráter aniônico do corante (Kimura et al.,2000). Além disso, devido ao fato de possuir dois grupos reativos (vinilsulfona e monoclorotriazina), pertence à classe de corantes reativos bifuncionais (Koprivanac et al., 2005).

Efluentes têxteis podem ser tratados por métodos biológicos, químicos ou físicos. Cada um destes métodos apresenta vantagens e desvantagens. Contudo, para o caso de efluentes contendo corantes, métodos biológicos e químicos não são indicados, uma vez que a maioria dos corantes sintéticos é estável a tais tratamentos (ÖZCAN et al., 2006), Além disso, apresentam algumas limitações. $\mathrm{O}$ tratamento biológico requer uma grande extensão de área e é limitado pela sensibilidade às variações diurnas, bem como pela toxicidade de alguns produtos químicos e também é menos flexível em projetos e operações (Crini, 2006). Os métodos químicos são geralmente dispendiosos e, na maioria dos casos, criam um problema de poluição secundária devido ao uso excessivo de produtos químicos e a acumulação de lodo concentrado (Immich et al., 2009). Em relação aos métodos físicos, a maior restrição está no fato de que os poluentes são somente transferidos para uma nova fase, ao invés de serem destruídos (Chernicharo et al., 2010). Entretanto, alguns materiais podem ser facilmente regenerados para serem novamente utilizados, como é o caso do adsorvente empregado neste estudo.

Dessa forma, um método físico que pode ser considerado bastante promissor para o tratamento de águas residuais é a adsorção, pois apresenta baixo custo, fácil operação, projeto simples, capacidade para tratar corantes em formas mais concentradas, bem como, elevada eficiência (Gök et al., 2010).

Neste sentido, a busca por adsorventes que apresentem estabilidade térmica e física, além de elevada capacidade de adsorção, deve ser constante. Considerando a variabilidade dos efluentes têxteis no que tange a parâmetros físico-químicos, tais como temperatura e $\mathrm{pH}$, este estudo teve como objetivo a avaliação do efeito do $\mathrm{pH}$ da solução, da temperatura do sistema e da granulometria das partículas de adsorvente na remoção do corante Azul Reativo $5 \mathrm{G}$ pelo adsorvente Dowex ${ }^{\mathrm{TM}}$ Optipore $^{\mathrm{TM}}$ SD-2.

\section{MATERIAIS E MÉTODOS}

Todos os experimentos foram realizados no Laboratório de Controle 
de Poluição (CP) do Departamento de Engenharia Química da Universidade Estadual do Oeste do Paraná - Unioeste Campus de Toledo.

\subsection{Materiais}

Neste trabalho foi utilizado o adsorvente Dowex ${ }^{\mathrm{TM}}$ Optipore ${ }^{\mathrm{TM}}$ SD-2, fabricado por Dow ${ }^{\circledR}$ Chemical Company e cedido pela empresa Coremal Comércio e Representações Maia Ltda, da cidade de Cotia - SP. Antes da sua utilização nos experimentos, o adsorvente foi seco em estufa (Cienlab) à $50^{\circ} \mathrm{C}$ por aproximadamente $6 \mathrm{~h}$, até obtenção de massa constante.

O corante Azul Reativo 5G utilizado neste estudo foi produzido e doado pela empresa Texpal Indústria Química S/A, de Valinhos - SP. Para a realização dos experimentos, foram preparadas soluções a partir da dissolução do corante em pó em água destilada. Foram preparadas soluções com diferentes concentrações, variando de acordo com o teste realizado.

\subsection{Procedimento Experimental}

Para a determinação do Ponto de Carga Zero $\left(\mathrm{pH}_{\mathrm{PCZ}}\right)$ do adsorvente, foi utilizado o método de titulação potenciométrica (Davranche et al., 2003). Para tanto, utilizou-se dois erlenmeyers contendo, cada um, inicialmente, $5 \mathrm{~g}$ de adsorvente e $100 \mathrm{~mL}$ de solução de $\mathrm{NaNO}_{3} \quad 0,1 \mathrm{~mol} \mathrm{~L}-1$. Titulou-se a solução de um erlenmeyer com $\mathrm{NaOH} 0,1$ mol $\mathrm{L}^{-1}$ e do outro com $\mathrm{HNO}_{3} \quad 0,1 \mathrm{~mol} \mathrm{~L}-$ 1. $\mathrm{O}$ valor do $\mathrm{pH}$ foi verificado antes e durante a titulação, anotando-se o volume da solução titulante que foi adicionado para cada alteração do $\mathrm{pH}$ até que este apresentasse pouca variação $\left( \pm 10^{-3}\right)$. A carga superficial líquida do adsorvente $\left({ }^{C}\right.$ ) foi obtida pela Equação 1 .

$c=\frac{C_{\text {Acido }}-C_{\text {Bass }}+\left[\mathrm{OH}^{-}\right]-\left[\mathrm{H}^{+}\right]}{C_{\text {Ads }}}$

Sendo $C_{\text {Acido }}$ e $C_{\text {Bare }}$ as concentrações de ácido e base (mol L1), respectivamente, $\left[\mathrm{OH}^{-}\right]$e $\left[\mathrm{H}^{+}\right]$as concentrações de equilíbrio dos íons básicos e ácidos, respectivamente $\left(\right.$ mol L $\left.\mathrm{L}^{-1}\right)$ e $C_{\text {Ads }}$ a concentração do adsorvente na suspensão $\left(\mathrm{g} \mathrm{L}^{-1}\right)$.

Para analisar a influência do $\mathrm{pH}$ da solução no processo de adsorção, preparou-se, separadamente, soluções de corante Azul Reativo 5G com $\mathrm{pH}$ variando de 1 a 12 e concentração inicial de $150 \mathrm{mg} \mathrm{L}^{-1}$. O pH foi ajustado utilizando-se soluções de $\mathrm{NaOH} 1 \mathrm{~mol} \mathrm{~L}^{-1}$ e $\mathrm{HCl} 1 \mathrm{~mol} \mathrm{~L}^{-1}$. Adicionou-se $50 \mathrm{~mL}$ de cada uma destas soluções em erlenmeyers contendo 0,005 $\mathrm{g}$ de adsorvente, deixando-as, em seguida, em incubadora refrigerada com agitação, também conhecida como shaker (Tecnal TE-424), sob agitação orbital à temperatura e rotação fixas de $30^{\circ} \mathrm{C}$ e $120 \mathrm{rpm}$, respectivamente, por um período de $96 \mathrm{~h}$. A determinação do tempo de equilíbrio de $96 \mathrm{~h}$ foi realizada previamente, a partir de um teste cinético.

Ao final deste teste, bem como dos testes para avaliação do efeito da temperatura e granulometria do adsorvente, retirou-se uma alíquota do sobrenadante e analisou-se a concentração final do corante presente na solução em espectrofotômetro UV-VIS (Shimadzu UV-1800). Os testes foram realizados em duplicata e a quantidade de corante Azul Reativo 5G adsorvido foi determinada a partir de um balanço de massa representado pela Equação 2 .

$$
q_{\theta q}=\frac{V_{S o t}\left(C_{A i}-C_{\theta q}\right)}{m_{a d s}}
$$

Sendo $q_{\curvearrowleft q}$ a concentração de equilíbrio do corante na fase sólida

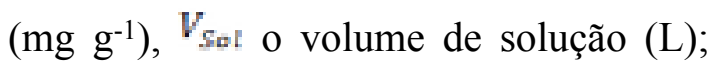
$C_{\text {Ai }}$ a concentração inicial de corante na solução $\left(\mathrm{mg} \mathrm{L}^{-1}\right){ }^{C^{C}}{ }$ a concentração do corante no equilíbrio $\left(\mathrm{mg} \mathrm{L}^{-1}\right) \mathrm{e}^{m_{c d s}}$ a massa de adsorvente em base seca (g).

$\mathrm{Na}$ análise do efeito da temperatura no processo de adsorção foram misturados $50 \mathrm{~mL}$ de solução de corante Azul Reativo 5G, com concentração inicial de $150 \mathrm{mg} \mathrm{L}^{-1}$, e $0,005 \mathrm{~g}$ de adsorvente em erlenmeyers 
de $125 \mathrm{~mL}$. Estes experimentos foram realizados na condição do $\mathrm{pH}$ que obteve maior taxa de remoção do corante $(\mathrm{pH} 2)$. As amostras foram deixadas em shaker sob agitação orbital de $120 \mathrm{rpm}$ durante $96 \mathrm{~h}$ e temperaturas de $20,30,40$ e $50{ }^{\circ} \mathrm{C}$, determinadas de forma aleatória.

A partir da análise do efeito da temperatura, avaliou-se a termodinâmica de adsorção do processo. Para tanto, foram avaliados três parâmetros: a variação da energia livre de Gibbs, da entalpia e da entropia do sistema.

A variação da energia livre de Gibbs é obtida a partir da Equação 3.

$$
\Delta G=-R T \ln \left(K_{\otimes q}\right)
$$

Sendo ${ }^{R}$ é a constante universal dos gases ideais $\left(8,314 \mathrm{~J} \mathrm{~mol}^{-1} \mathrm{~K}^{-1}\right),{ }^{T}$ a temperatura de operação do sistema $(\mathrm{K})$ e $K_{e q}$ a constante de equilíbrio de adsorção $\left(\mathrm{L} \mathrm{g} \mathrm{g}^{-1}\right)$, obtida pela Equação 4 .

$$
K_{\sigma q}=\frac{\left(C_{A i}-C_{\sigma Q}\right)}{C_{e q}}
$$

As variações de entalpia e entropia do sistema são obtidas a partir de um gráfico de ${ }^{\Delta G}$ em função de ${ }^{T}$, onde, de acordo com a Equação 5, a interseção e inclinação de uma reta com o eixo das ordenadas fornece os valores de ${ }^{\Delta H} \mathrm{e}^{\Delta S}$, respectivamente.

$$
\Delta G=\Delta H-T \Delta S
$$

Para analisar a influência do diâmetro das partículas de adsorvente no processo, primeiramente, avaliou-se a distribuição granulométrica do material. Para tanto, $35 \mathrm{~g}$ de adsorvente foram submetidos a um peneiramento por meio de um conjunto de peneiras da série Tyler (Bertel) com as seguintes aberturas das malhas: 14, 28, 32 e 48 mesh, além do fundo da peneira, que possui abertura de 200 mesh. O diâmetro médio das partículas de adsorvente foi representado pelo modelo do diâmetro médio de Sauter $(\bar{D})$, descrito pela Equação 6.

$$
\bar{D}=\frac{1}{\sum\left(X / \overline{d_{p}}\right)}
$$

Sendo ${ }^{X}$ a fração mássica das partículas retidas em cada peneira e $\bar{d}_{p}$ o diâmetro médio das partículas, obtido a partir da média aritmética da abertura da malha das peneiras entre as quais o material ficou retido.

Para realização dos experimentos, escolheu-se três faixas granulométricas, mostradas na Tabela 1 .

Tabela 1. Faixas granulométricas utilizadas na avaliação do efeito do diâmetro das partículas na adsorção

\begin{tabular}{cc}
\hline $\begin{array}{c}\text { Faixa } \\
\text { granulométrica } \\
(\mathrm{cm})\end{array}$ & $\begin{array}{c}\text { Diâmetro médio } \\
\text { das partículas } \\
(\mathrm{cm})\end{array}$ \\
\hline $0,01<d_{p}<0,12$ & 0,063 (Mistura) \\
$0,05<d_{p}<0,06$ & 0,055 \\
$0,06<d_{p}<0,12$ & 0,090 \\
\hline
\end{tabular}

A escolha de tais faixas foi baseada no seguinte critério: primeiramente, escolheu-se não trabalhar com partículas finas, que possuem diâmetros inferiores a $0,05 \mathrm{~cm}$; em seguida, optou-se por trabalhar com as duas faixas granulométricas que apresentaram a maior porcentagem de massa retida, correspondentes aos diâmetros médios de partícula de 0,055 $\mathrm{cm}$ e $0,090 \mathrm{~cm}$; finalmente, utilizouse a mistura granulométrica de todos os diâmetros de partícula $\left(\overline{d_{p}}=0,063 \mathrm{~cm}\right)$.

Para cada faixa granulométrica, $0,005 \mathrm{~g}$ de adsorvente foram colocados em contato com $50 \mathrm{~mL}$ de solução de corante Azul Reativo 5G com concentração inicial de $150 \mathrm{mg} \mathrm{L}^{-1}$. Este experimento foi realizado na condição do $\mathrm{pH}$ e temperatura em que se obteve maior remoção do corante $(\mathrm{pH} 2$ e temperatura de $30{ }^{\circ} \mathrm{C}$ ) e as amostras foram deixadas em shaker sob agitação orbital de 120 rpm durante $96 \mathrm{~h}$. 


\section{RESULTADOS E DISCUSSÃO}

\subsection{Determinação do ponto de carga zero do adsorvente}

Os adsorventes, em geral, desenvolvem cargas na interface sólidolíquido devido à dissociação ou adsorção de íons da solução. A caracterização dessas cargas é importante no que se refere às aplicações dos materiais como adsorventes. A faixa de $\mathrm{pH}$ em que as cargas superficiais líquidas do adsorvente são nulas é referida como Ponto de Carga Zero $\left(\mathrm{pH}_{\mathrm{PCZ}}\right)$ do adsorvente.

$\mathrm{O}$ valor do $\mathrm{pH}_{\mathrm{PCZ}}$ do adsorvente Dowex $^{\mathrm{TM}}$ Optipore ${ }^{\mathrm{TM}}$ SD-2 foi obtido a partir da construção de um gráfico da carga superficial líquida do adsorvente em função do $\mathrm{pH}$, conforme apresentado na Figura 1.

Analisando a Figura 1, observase que a carga superficial líquida do adsorvente decresce com o aumento do $\mathrm{pH}$. Na faixa de $\mathrm{pH}$ entre 4 e 10, a carga superficial do adsorvente é nula. Esta faixa é definida como o $\mathrm{pH}_{\mathrm{PCZ}}$ do adsorvente. A adsorção de cátions é favorecida a um $\mathrm{pH}$ maior que o $\mathrm{pH}_{\mathrm{PCZ}}$, pois, nesse caso, a carga líquida da superfície do adsorvente é negativa $(\mathrm{pH}>$ 10), enquanto que a adsorção de ânions é favorecida a um $\mathrm{pH}$ menor que o $\mathrm{pH}_{\mathrm{PCZ}}$, uma vez que, dessa forma, a carga líquida da superfície do adsorvente é positiva ( $\mathrm{pH}<4)$ (Tagliaferro et al., 2011).

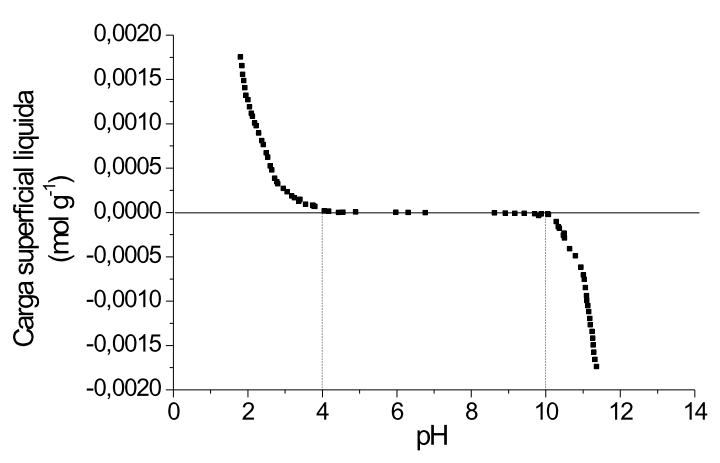

Figura 1. Carga superficial líquida do adsorvente Dowex ${ }^{\mathrm{TM}}$ Optipore ${ }^{\mathrm{TM}} \mathrm{SD}-2$ em função do $\mathrm{pH}$.
Borba et al. (2009) obtiveram resultado semelhante $\left(4<\mathrm{pH}_{\mathrm{PCZ}}<11\right)$ em sua pesquisa sobre o processo de troca iônica do sistema multicomponente cobre-zinco-sódio utilizando a resina Amberlite IR 120, que possui grupo funcional sulfônico $\left(-\mathrm{SO}_{3} \mathrm{H}\right)$.

Pereira et al. (2001), investigaram a adsorção de corantes aniônicos utilizando carvões básicos. $\mathrm{O}$ adsorvente $\left(8,4<\mathrm{pH}_{\mathrm{PCZ}}<10\right)$ foi posto em contato com soluções de corante com $\mathrm{pH}$ entre 2,7 e 12. Para as soluções com pH ácido, o adsorvente removeu o corante mais eficientemente, enquanto em $\mathrm{pH}$ básico, a adsorção foi prejudicada, pois, neste caso, existem forças de repulsão eletrostáticas entre o corante e a superfície negativa do carvão.

\subsection{Efeito do pH da solução}

Segundo Mall et al. (2006), alterações no $\mathrm{pH}$ podem afetar o processo de adsorção por meio da dissociação de grupos funcionais presentes nos sítios ativos do adsorvente. Dessa forma, é necessário conhecer a influência que este parâmetro exerce sobre o processo de adsorção de corantes.

$\mathrm{Na}$ Figura 2 são apresentados os valores da quantidade de corante adsorvida em função do $\mathrm{pH}$ da solução.

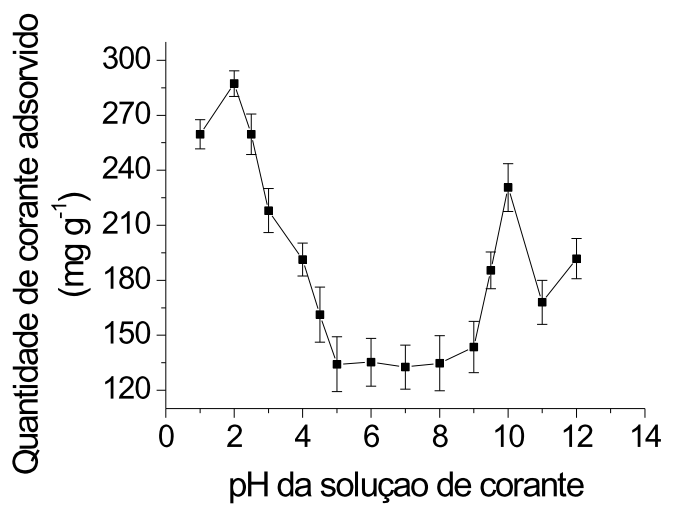

Figura 2. Quantidade de corante adsorvida em função do $\mathrm{pH}$ da solução $c^{C_{0}}=150 \mathrm{mg} \mathrm{L}^{-1},{ }^{m_{a d s}}=0,005 \mathrm{~g},{ }^{d_{p}}=$ mistura, ${ }^{T}=30{ }^{\circ} \mathrm{C}$, Agitação $=120 \mathrm{rpm}$ ).

Os resultados mostram que, nas condições do experimento, a maior quantidade de corante adsorvido $(292 \pm 8$ 
$\mathrm{mg} \mathrm{g}^{-1}$ ) foi obtida para a solução com $\mathrm{pH}$ igual a 2, enquanto a menor quantidade adsorvida (aproximadamente $134 \pm 9 \mathrm{mg}$ $\mathrm{g}^{-1}$ ) foi observada para as solução com pH entre 5 a 9.

O corante utilizado neste estudo possui um grupo sulfonato, responsável por seu caráter aniônico, possuindo, portanto, carga superficial negativa. De acordo com a análise do $\mathrm{pH}_{\mathrm{PCZ}}$, tem-se que em meio ácido $(\mathrm{pH}<4)$ a superfície do adsorvente é carregada positivamente, sendo assim, segundo AlDegs et al. (2000), como a superfície do adsorvente possui cargas positivas em $\mathrm{pH}$ menores que 4, existe uma alta atração eletrostática entre a superfície positivamente carregada do adsorvente e o corante aniônico, o que explica o fato da adsorção do corante ser favorecida em pH inferior a 4.

O resultado obtido condiz com o encontrado por Martins et al. (2013), Fiorentin et al. (2010) e Fagundes-Klen et al. (2012), quando pesquisaram a adsorção do mesmo corante utilizando carvão ativado, bagaço de laranja e a macrófita Salvínia Sp., respectivamente. Por outro lado, no estudo realizado por Borges (2006), que investigou a adsorção do corante Azul Reativo $5 \mathrm{G}$ em sistema batelada e em leito fixo utilizando como adsorvente o carvão ativado, os resultados mostraram que o $\mathrm{pH} \mathrm{7,25}$ foi o mais favorável à adsorção. Além disso, na pesquisa realizada por Módenes et al. (2011) sobre a utilização da macrófita Egeria Densa na biossorção do corante Azul Reativo 5G em processo batelada as melhores condições de operação foram obtidas para $\mathrm{pH}$ igual a 1 . Dessa forma, pode-se afirmar que a melhor condição de $\mathrm{pH}$ da solução, quando analisadas para o mesmo corante, depende do tipo de adsorvente empregado no processo.

\subsection{Efeito da temperatura}

A temperatura é um dos parâmetros que mais influenciam nas relações de equilíbrio, uma vez que afeta a agitação das moléculas do sistema, interferindo também nas forças de atração e repulsão entre o adsorvato e o adsorvente. Os resultados obtidos para a concentração de equilíbrio do corante na fase sólida em diferentes temperaturas são apresentados na Tabela 2.

Tabela 2. Capacidade de adsorção em cada temperatura $\left({ }^{C_{0}}=150 \mathrm{mg} \mathrm{L}^{-1}\right.$,

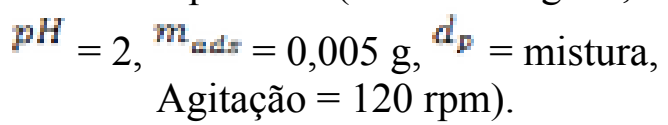

\begin{tabular}{cc}
\hline Temperatura $\left({ }^{\circ} \mathrm{C}\right)$ & $q_{\text {ब्q }}\left(\mathrm{mg} \mathrm{g}^{-1}\right)$ \\
\hline 20 & $205 \pm 5$ \\
30 & $235 \pm 11$ \\
40 & $216 \pm 7$ \\
50 & $200 \pm 9$ \\
\hline
\end{tabular}

Analisando os dados apresentados na Tabela 2, observa-se que, na faixa de temperatura e condições estudadas, para temperaturas acima de $30^{\circ} \mathrm{C}$, um aumento neste parâmetro resulta em um decréscimo na quantidade de corante adsorvido. Por outro lado, quando a temperatura é diminuída para $20^{\circ} \mathrm{C}$, a capacidade de adsorção do corante foi menor. Dessa forma, a quantidade máxima removida pelo adsorvente $(235 \pm$ $11 \mathrm{mg} \mathrm{g}^{-1}$ ) ocorreu em $30^{\circ} \mathrm{C}$.

Martins et al. (2013) avaliaram as temperaturas de 30,45 e $60^{\circ} \mathrm{C}$ na adsorção do corante Azul Reativo 5G em carvão ativado e observaram que o melhor resultado foi obtido em $30^{\circ} \mathrm{C}$. Lambrecht et al. (2009) pesquisaram a adsorção do mesmo corante utilizando carvão ativado e xisto pirolisado nas temperaturas de 30 e $40^{\circ} \mathrm{C}$ e observaram que, para o processo com ambos os adsorventes, a melhor remoção do corante foi obtida para a temperatura de $30{ }^{\circ} \mathrm{C}$.

A estimativa dos parâmetros termodinâmicos é feita pela caracterização do processo de adsorção de um estado inicial a um estado final de equilíbrio e pode determinar se o processo é espontâneo, endo ou exotérmico e o grau de afinidade entre adsorvente e adsorvato. Além disso, tais parâmetros podem fornecer informações 
sobre a heterogeneidade da superfície do adsorvente e se o processo envolve adsorção física ou química (Calvet, 1989). Os valores dos parâmetros termodinâmicos de adsorção são apresentados na Tabela 3 .

Pela análise dos resultados, observa-se que o valor obtido para a variação da entalpia é negativo, o que sugere que o processo é exotérmico. $\mathrm{O}$ fato de o valor de ${ }^{\Delta H}$ ser menor que $40 \mathrm{~kJ} \mathrm{~mol}^{-1}$, indica que o processo de adsorção é de natureza física (fisissorção), com ligações fracas de Van der Waals entre o corante e o adsorvente.

Tabela 3. Parâmetros termodinâmicos da adsorção do corante Azul Reativo 5G.

\begin{tabular}{cccc}
\hline$T$ & $\Delta G$ & $\Delta H$ & $\Delta S$ \\
$(\mathrm{~K})$ & $\left(\mathrm{kJ} \mathrm{mol}^{-1}\right)$ & $\left(\mathrm{kJ} \mathrm{mol}^{-1}\right)$ & $\left(\mathrm{kJ} \mathrm{mol}^{-1} \mathrm{~K}^{-1}\right)$ \\
\hline 293 & $-1,21$ & & \\
303 & $-1,74$ & & \\
313 & $-1,42$ & $-3,43$ & $-0,007$ \\
323 & $-1,09$ & & \\
\hline
\end{tabular}

A variação de entropia está relacionada às variações de ordemdesordem de um sistema, de modo que, quanto mais randômico for o sistema, maior a sua entropia. $\mathrm{O}$ valor negativo da variação de entropia indica um aumento no grau de organização do sistema, associado à acomodação das partículas de corante em camadas mais ordenadas na superfície do adsorvente. Esse aumento é compensado pela liberação de energia representada pela variação de entalpia, garantindo que o processo global é termodinamicamente coerente. A equação da energia livre de Gibbs em termos de $\Delta H$ e ${ }^{\Delta S}$ evidencia isso, uma vez que $\Delta G$ deve ser sempre negativo para que um processo seja termodinamicamente favorável.

Resultados semelhantes foram obtidos por Errais, et al. (2011) e Elkady et al. (2011) em suas pesquisas sobre a adsorção dos corantes reativos Vermelho 120 em argila in natura e Vermelho Remazol 198 em casca de ovo, respectivamente. Ambos obtiveram que o processo de adsorção utilizando os respectivos adsorventes é um processo exotérmico, espontâneo e possui afinidade entre adsorvente/ adsorvato.

\subsection{Efeito do diâmetro das partículas de adsorvente}

A avaliação da distribuição granulométrica das partículas de adsorvente foi realizada por peneiramento. $\mathrm{O}$ diâmetro médio das partículas, a massa e a fração mássica das partículas de adsorvente, retidas em cada peneira são apresentadas na Tabela 4.

Tabela 4. Distribuição granulométrica das partículas de adsorvente.

\begin{tabular}{|c|c|c|c|c|}
\hline $\begin{array}{l}\text { Abertura } \\
\text { da malha } \\
(\mathrm{mm})\end{array}$ & $\begin{array}{l}\overline{d_{p}} \\
(\mathrm{~cm})\end{array}$ & $\begin{array}{c}\text { Massa } \\
\text { retida } \\
(\mathrm{g})\end{array}$ & $\begin{array}{c}\text { Massa } \\
\text { retida } \\
(\%)\end{array}$ & $X$ \\
\hline 1,190 & 0,111 & 6,984 & 19,954 & 0,200 \\
\hline 0,590 & 0,090 & 9,543 & 27,266 & 0,273 \\
\hline 0,500 & 0,055 & 11,176 & 31,931 & 0,320 \\
\hline 0,297 & 0,040 & 7,297 & 20,849 & 0,208 \\
\hline 0,210 & 0,026 & 0 & 0 & 0 \\
\hline \multicolumn{2}{|c|}{ Total } & 35 & 100 & 1 \\
\hline
\end{tabular}

A partir dos resultados apresentados na Tabela 3 e utilizando a Equação 6, o diâmetro médio de Sauter encontrado para a mistura granulométrica foi igual a $0,063 \mathrm{~cm}$.

As três faixas granulométricas escolhidas para avaliação do diâmetro e os resultados obtidos para a concentração de equilíbrio do corante na fase sólida em cada faixa são apresentados na Tabela 5. 
Tabela 5. Capacidade de adsorção para cada diâmetro de partícula $\left({ }^{C_{0}}=150 \mathrm{mg}\right.$ $\mathrm{L}^{-1},{ }^{p H}=2,{ }^{m_{\text {eds }}}=0,005 \mathrm{~g},{ }^{T}=30^{\circ} \mathrm{C}$, Agitação $=120 \mathrm{rpm}$ ).

\begin{tabular}{ccc}
\hline $\begin{array}{c}\text { Faixa } \\
\text { granulométrica }\end{array}$ & $\begin{array}{c}\bar{d}_{p} \\
(\mathrm{~cm})\end{array}$ & $\begin{array}{c}q_{\text {eq }} \\
\left(\mathrm{mg} \mathrm{g}^{-1}\right)\end{array}$ \\
\hline $0,01<d_{p}<0,12$ & $\begin{array}{c}0,06 \\
3\end{array}$ & $230 \pm 6$ \\
$0,05<d_{p}<0,06$ & 0,05 & $169 \pm 2$ \\
$0,06<d_{p}<0,12$ & 0,09 & $115 \pm 3$ \\
& 0 & \\
& & \\
0
\end{tabular}

Conforme observa-se na Tabela 4, a mistura granulométrica forneceu a maior capacidade de adsorção do corante Azul Reativo 5G, visto que a presença de partículas com diâmetros inferiores aos encontrados nas demais faixas, contribui para que a mistura apresente superfície de contato entre o adsorvente e a solução de corante maior que as demais. Da mesma forma, partículas maiores, com diâmetro médio de 0,090 cm, apresentaram a menor capacidade de retenção do corante devido à sua menor superfície de contato.

Menezes (2010) em sua pesquisa sobre a remoção do corante Azul Reativo 5G a partir de soluções aquosas utilizando o bagaço do maracujá amarelo em sistema batelada, analisou a influência da granulometria das partículas adsorventes e concluiu que, dentre as granulometrias estudadas $(0,050 \mathrm{~cm}$, $0,025 \mathrm{~cm}$ e $0,011 \mathrm{~mm}$ ) o menor diâmetro de partícula também foi o que apresentou a maior remoção de corante.

A avaliação da influência destes parâmetros é realizada afim de que os dados obtidos em laboratório possam ser passados para a escala industrial, diminuindo, consequentemente, os custos de um projeto (Borba et al., 2011). Industrialmente, o processo de adsorção é, na maioria dos casos, operado em coluna de leito fixo, visto que, neste modo de operação é possível tratar maiores volumes de efluentes.

\section{CONCLUSÃO}

Neste trabalho avaliou-se o efeito do $\mathrm{pH}$ da solução, da temperatura do sistema e da granulometria das partículas de adsorvente na remoção do corante Azul Reativo 5G pelo adsorvente Dowex $^{\mathrm{TM}}$ Optipore ${ }^{\mathrm{TM}}$ SD-2.

Os resultados obtidos demonstram que: o $\mathrm{pH}_{\mathrm{PCZ}}$ do adsorvente ficou entre 4 e 10; a solução com $\mathrm{pH} 2$ foi a que apresentou a maior quantidade de corante adsorvido $\left({ }^{q_{\text {eq }}}=292 \pm 8 \mathrm{mg} \mathrm{g}^{-1}\right)$; nas condições de temperatura estudadas, a maior quantidade de corante removida pelo adsorvente $\left(^{q \in q}=235 \pm 11 \mathrm{mg} \mathrm{g}^{-}\right.$ $\left.{ }^{1}\right)$ foi obtida em $30^{\circ} \mathrm{C}$, além disso, os parâmetros termodinâmicos indicam que o processo é exotérmico, espontâneo e possui afinidade entre adsorvente e adsorvato; na análise da granulometria das partículas, os melhores resultados $\left({ }^{q_{\text {eq }}}=230 \pm 6 \mathrm{mg} \mathrm{g}^{-1}\right)$ foram obtidos para a mistura granulométrica.

Através dos resultados obtidos neste trabalho, conclui-se que o adsorvente Dowex ${ }^{\mathrm{TM}}$ Optipore ${ }^{\mathrm{TM}}$ SD2 foi eficiente na remoção do corante Azul Reativo 5G, apresentando elevada capacidade de adsorção (superior a 230 $\mathrm{mg} \mathrm{g}^{-1}$ ) quando comparado a outros tipos de adsorventes. Desta forma, sugere-se que tal adsorvente pode ser utilizado com elevada eficiência na remoção do corante Azul Reativo 5G de efluentes têxteis.

\section{REFERÊNCIAS}

AL-DEGS, Y.; KHRAISHEH, M.A.M.; ALLEN, S.J.; AHMAD, M. N. 2000. Effect of carbon surface chemistry on the removal of reactive dyes from textile effluent. Water Research. Vol 34, p. $927-$ 935.

ALKAN, M.; DOGAN, M.; TURHAN, Y.; DEMIRBAS, O.; TURAN, P. 2008. Adsorption kinetics and mechanism of maxilon blue $5 \mathrm{G}$ dye on sepiolite from aqueous solutions. Chemical Engineering Journal. Vol 139, p. 213-223. 
BORBA, C. E. 2009. Estudo do processo de troca ionica do sistema multicomponente cobre-zinco utilizando resina Amberlite IR 120. Tese de doutorado em engenharia química. Universidade Estadual de Campinas. Campinas, SP.

BORBA, C. E.; SILVA, E. A.; SPOHR, S.; SANTOS, G. H. F.; GUIRARDELLO, R. 2011. Application ofthe mass action law to describe ion exchange equilibrium in a fixed-bed column. Chemical Engineering Journal. Vol. 172, p. 312-320.

BORGES, E. R. 2006. Remoção do corante reativo azul $5 G$ através de processo de separação por membranas $e$ adsorção com carvão ativado. Dissertação de mestrado em engenharia química. Universidade Estadual de Maringá. Maringá, PR.

CALVET, R. 1989. Adsorption of organic chemicals in soils. Environmental Health Perspectives. Vol 83, p. 145-177.

CHERNICHARO, C. A. DE L.; STUETZ, R. M; SOUZA, C. L.; MELO, G. C. B. de. 2010. Alternativas para o controle de emissões odorantes em reatores anaeróbios tratando esgoto doméstico. Engenharia Sanitária e Ambiental. Vol 15, p. 229-236.

CRINI, G. 2006. Non-conventional low-cost adsorbents for dye removal: A review. Bioresource Technology. Vol 97, p. 1061-1085.

DAVRANCHE M; LACOUR S; BORDAS F. 2003. An easy determination of the surface chemical properties of simple and natural solids. Journal of Chemical Education. Vol. 80, p. 76-78.

ELKADY, M. F.; ILBRAHIM, A. M.; EL-LATIF, M. M. A. 2011. Assessment of the adsorption kinetics, equilibrium and thermodynamic for the potential removal of reactive red dye using eggshell biocomposite beads. Desalination. Vol. 278, p. 412-423.

ERRAIS E.; DUPLAY J.; DARRAGI F.; M'RABET I.; AUBERT A.; HUBER F.; MORVAN G. 2011. Efficient anionic dye adsorption on natural untreated clay: Kinetic study and thermodynamic parameters. Desalination. Vol. 275, p. 74-81.

FAGUNDES-KLEN, $\quad$ M. R.; CERVELIN, P. C.; VEIT, M. T.; GONÇALVES, G. DA C.; BERGAMASCO R.; SILVA, F. V. DA. 2012. Adsorption kinetics of blue $5 \mathrm{~g}$ dye from aqueous solution on dead floating aquatic macrophyte: effect of $\mathrm{pH}$, temperature, and pretreatment. Water Air Soil Pollut. Vol. 223, p. 4369-4381.

FIORENTIN, L. D. F.; TRIGUEROS, D. E. G.; MÓDENES, A. N.; ESPINOZAQUIÑONES, F. R.; PEREIRA, N. C.; BARROS, S. T. D.; SANTOS, O. A. A. 2010. Biosorption of reactive blue $5 \mathrm{G}$ dye onto drying orange bagasse in batch system: Kinetic and equilibrium modeling. Chemical Engineering Journal. Vol. 163, p. 68-77.

GÖK, O.; ÖZCAN, A. S.; ÖZCAN, A. 2010. Adsorption behavior of a textile dye of Reactive Blue 19 from aqueous solutions onto modified bentonite. Applied Surface Science. Vol 256, p. 5439-5443.

IMMICH, A. P. S; SOUZA, A. A. U. DE; SOUZA, S. M. DE A. G. U. de. 2009. Removal of remazol blue RR dye from aqueous solutions with neem leaves and evaluation of their acute toxicity with Daphnia Magna. Journal of Hazardous Materials. Vol. 164, p. 1580-1585.

KHADHRAOUI, M.; TRABELSI, H.; KSIBI, M.; BOUGUERRA, S.; ELLEUCH, B. 2009. Discoloration and detoxicification of a Congo red dye solution by means of ozone treatment for a possible water reuse. Journal of Hazardous Materials. Vol 161, p. 974 981.

KIMURA I. Y.; FÁVERE V. T. DE; LARANJEIRA M. C. M.; JOSUÉ A.; NASCIMENTO A. Do. 2000. Avaliação da capacidade de adsorção do corante reativo laranja 16 pela quitosana. Acta Scientiarum. Vol 22, p. 1161-1166.

KOPRIVANAC, N.; KUSIC, H.; VUJEVIC, D.; PETERNEL, I.; LOCKE, 
B. R. 2005. Influence of iron on degradation of organic dyes in corona. Journal of Hazardous Materials. Vol. 117, p. 113-119.

LAMBRECHT, R.; BARROS, M. A. S. D.; COSSICH, E. S.; SILVA, E. A.; MATTA, G. K. L.; STACHIW, R. 2009. Adsorption of reactive blue $5 \mathrm{G}$ dye by activated carbon and pyrolyzed shale oil residue. Journal Adsorption Science e Technology. Vol. 25, p. 741-749.

MALL, I. D.; SRIVASTAVA, V. C.; AGARWAL, N. K. 2006. Removal of Orange-G and Methyl Violet dyes by adsorption onto bagasse fly ash - kinetic study and equilibrium isotherm analyses. Dyes and Pigments. Vol 69, p. 210-223.

MARTINS, T. D.; SCHIMMEL, D.; SANTOS, J. B. O. DOS; SILVA, E. A. da. 2013. Reactive Blue 5G Adsorption onto Activated Carbon: Kinetics and Equilibrium. Journal of Chemical \& Engineering Data. Vol. 58, p. 106-114.

MENEZES, M. L. DE. 2010. Remoção de corante reativo azul $5 \mathrm{G}$ a partir de soluções aquosas utilizando o bagaço do maracujá amarelo como adsorvente. Dissertação de mestrado em engenharia química. Universidade Estadual de Maringá. Maringá, PR.

MÓDENES, A. N.; ESPINOZAQUIÑONES, F. R.; ALFLEN, V. L.; COLOMBO, A.; BORBA, C. E. 2011. Utilização da macrófita egeria densa na biosorção do corante reativo $5 \mathrm{G}$. Engevista. Vol. 13, p. 160-166.

ÖZCAN, A.; ÖNCÜ, E. M.; ÖZCAN, A. S. 2006. Adsorption of Acid Blue 193 from aqueous solutions onto DEDMA-sepiolite. Journal of Hazardous Materials. Vol 129, p. 244-252.

PEREIRA, M. F. R., ÓRFÃO, J. J. M., FIGUEIREDO, J. L. 2001. Remoção da cor em efluentes da indústria têxtil por adsorção em carvões ativados modificados. Adsorbentes en la solución de algunos problemas ambientales. Editora Cyted. Madri.

TAGLIAFERRO, G.V; PEREDIRA, P. H. F.; RODRIGUES, L. A.; SILVA, M. L. C. P. 2011. Adsorção de chumbo, cádmio e prata em óxido de nióbio (V) hidratado preparado pelo método da precipitação em solução homogênea. Química Nova. Vol. 34, p. 101-105.

Texpal Indústria Química. 2005. Boletim técnico do corante Azul Reativo BF $5 G$. Valinhos, SP.

Tríplice Cor: Corantes e pigmentos. 2011. Disponível em: < http:// www.triplicecor.com.br/corantes/dicas/ substancias-soluveis-corantes $>$ [Acesso em: 13 de setembro de 2011] 